\section{Optimizing bupivacaine dosing for Cesarean delivery - II}

\section{To the Editor:}

We read with interest the study by Bryson et al. ${ }^{1}$ comparing a small dose $(4.5 \mathrm{mg})$ of spinal bupivacaine with a more standard dose $(12 \mathrm{mg})$ on maternal hemodynamics for Cesarean delivery. Patients in both groups received intrathecal fentanyl $50 \mu \mathrm{g}$, and we were surprised to read the authors' view that administering such a relatively large dose is supported by the current literature. Since an intrathecally administered fentanyl dose of $50 \mu \mathrm{g}$ is obviously at the upper limit of reported recommendations, we believe further commentary is warranted, as most recently-published studies used intrathecal fentanyl doses higher than indicated by dose-response studies. While a Letter to the Editor does not permit detailed reporting of the literature, recent publications suggest the optimal dose of subarachnoid fentanyl for Cesarean delivery should be between 20-25 $\mu \mathrm{g}$. Even these doses may be excessive, as Hunt et al. ${ }^{2}$ suggest that the optimum dose of intrathecal fentanyl should be $6.25 \mu \mathrm{g}$, and that larger doses confer no additional benefit, while resulting in more opioid-related side effects. Although this latter study was not well powered, its results have since been confirmed. ${ }^{3}$ Belzarena et al. ${ }^{4}$ found that maternal pruritus occurs with intrathecal fentanyl at doses exceeding $20 \mu \mathrm{g}$. Fentanyl $10 \mu \mathrm{g}$ also increases the quality of analgesia when spinal ropivacaine is used for Cesarean delivery, without increasing the incidence of side effects. In non-obstetrical patients, there is no evidence that doses larger than $10 \mu \mathrm{g}$ are beneficial. In their discussion, Bryson et al. ${ }^{1}$ state that doses of up to $60 \mu \mathrm{g}$ of intrathecal fentanyl have been reported. However, only one trial reported on the effects of $60 \mu \mathrm{g}$ of intrathecal fentanyl; ${ }^{4}$ while, in other trials, it has been shown that increasing the dose of fentanyl above $10 \mu \mathrm{g}$ increases the duration of postoperative analgesia. ${ }^{4}$ Nevertheless, a modest prolongation in the duration of postoperative analgesia is of uncertain importance for fentanyl, in view of the drug's relatively short duration of action. If the goal is to increase the duration of postoperative analgesia, then intrathecal morphine is a better choice. ${ }^{3}$ Moreover, excessive increases in dose of intrathecal fentanyl may be associated with profound ventilatory depression.

Early trials using fentanyl with a combined spinalepidural (CSE) technique most often reported use of $25 \mu \mathrm{g}$ intrathecally. This dose remains in common use in many maternity units, despite the fact that doseresponse studies have identified that the fentanyl intra- thecal $\mathrm{ED}_{95}$ is approximately $15 \mu \mathrm{g}$, when used alone, or when combined with a local anesthetic. ${ }^{5}$ Moreover, when very large doses are used for CSE during labour, side effects, including uterine hyperactivity and non-reassuring fetal heart rate tracings may occur. Therefore, we recommend caution regarding the dose selection of intrathecal fentanyl in obstetric anesthesia, and suggest doses in excess of $15 \mu \mathrm{g}$ be avoided for both Cesarean delivery and labour analgesia.

Marie Bruyère MD

Dan Benhamou MD

Hôpital Antoine Béclère, Clamart, France

E-mail: dan.benhamou@bct.aphp.fr

Accepted for publication October 24, 2007.

\section{References}

1 Bryson GL, MacNeil R, Jeyaraj LM, Rosaeg OP. Small dose spinal bupivacaine for cesarean delivery does not reduce hypotension but accelerates motor recovery. Can J Anesth 2007; 54: 531-7.

2 Hunt CO, Naulty JS, Bader AM, et al. Perioperative analgesia with subarachnoid fentanyl-bupivacaine for cesarean delivery. Anesthesiology 1989; 71: 535-40.

3 Dahl JB, Jeppesen IS, Jorgensen H, Wetterslev J, Moiniche $S$. Intraoperative and postoperative analgesic efficacy and adverse effects of intrathecal opioids in patients undergoing cesarean section with spinal anesthesia: a qualitative and quantitative systematic review of randomized controlled trials. Anesthesiology 1999; 91: 1919-27.

4 Belzarena SD. Clinical effects of intrathecally administered fentanyl in patients undergoing cesarean section. Anesth Analg 1992; 74: 653-7.

5 Wong CA, Scavone BM, Slavenas JP, et al. Efficacy and side effect profile of varying doses of intrathecal fentanyl added to bupivacaine for labor analgesia. Int J Obstet Anesth 2004; 13: 19-24.

\section{Reply:}

We thank Drs. Tinits, Bruyere and Benhamou for their interest in our article and for their thoughtful comments.

Regarding Dr. Tinits' comments about baricity and the cephalad extension of our sensory blocks, we would first like to correct Dr. Tinits' assertion that our solutions were mixed in water. The second paragraph of our methods indicates that saline was used as the diluent. Regardless, the relative impact of saline or water on the baricity of the final solution is relatively minor. 
Baricity is the density of the local anesthetic solution divided by the density of cerebrospinal fluid (CSF). Research from the Ottawa Hospital cited by Dr. Tinits defined the densities of CSF, local anesthetic solutions, and common additives. ${ }^{1}$ The density of CSF in the parturient at term is $1.00033 \pm 0.00010 \mathrm{~g} \cdot \mathrm{mL}^{-1}$, while the densities of water and saline are 0.9933 and 0.9995 $g \cdot m L^{-1}$ respectively. The densities of isobaric bupivacaine, fentanyl, and saline solutions are $0.9993 \mathrm{~g} \cdot \mathrm{mL}^{-1}$, 0.9932 and $0.9995 \mathrm{~g} \cdot \mathrm{mL}^{-1}$, respectively. While we did not directly measure the density of the solutions used in this study, the components of the $4.5 \mathrm{mg}$ bupivacaine solution are relatively hypobaric when compared to CSF, but range within $0.0003 \mathrm{~g} \cdot \mathrm{mL}^{-1}$ of solutions considered isobaric by clinicians. As such, it would seem unlikely that a strongly hypobaric solution was responsible for the observed cephalad extension of anesthesia.

With regards to the use of tetracaine, a $1 \%$ solution in saline has a specific gravity of $0.9995 \mathrm{~g} \cdot \mathrm{mL}^{-1}$. Given the relatively small difference in density between saline and water noted above, a $1 \%$ tetracaine solution in water could be expected to be of a similar density to the solutions employed in our study. We cannot comment directly on the use of tetracaine for Cesarean delivery, but our anecdotal experience suggests that traditional doses of 12 to $15 \mathrm{mg}$ are associated with a higher frequency of hypotensive events and deeper levels of motor block than with bupivacaine. Continuous spinal-epidural anesthesia is, indeed, a useful technique but was not the subject of the present paper.

With respect to the comments by Drs. Bruyere and Benhamou, we recognize that lower doses of fentanyl have been advocated for both Cesarean and labour analgesia. During the design phase of our trial, we were influenced by the $6 \%$ rate of analgesic supplementation reported in Ben-David's study that used $25 \mu \mathrm{g}$ doses of fentanyl. ${ }^{2}$ As there was evidence supporting the use of larger doses of fentanyl, ${ }^{3,4}$ we opted to proceed with the $50 \mathrm{\mu g}$ dose. The results of our study suggest this larger dose was not associated with poor neonatal outcomes and, while not reported in the manuscript, we found no evidence of maternal respiratory depression or naloxone use in our cohort. Subjects receiving the $50 \mathrm{ug}$ dose of fentanyl in either arm of our study preferred the present technique to their previous Cesarean delivery. In the spirit of continuous quality improvement, we have re-evaluated our dose of opioid in light of the high rate of pruritus and nausea reported in the present study. Our current clinical practice is to administer 5-mg isobaric bupivacaine combined with fentanyl 10-15 $\mathrm{Mg}$ and morphine 100-150 $\mathrm{ug}$ intrathecally. We have yet to formally assess the impact of this change on side effects, but we have not observed an increased requirement for postoperative analgesic supplementation.
Robert MacNeil MD FRCPC

Gregory L. Bryson MD FRCPC MSC

The Ottawa Hospital - Civic Campus, Ottawa,

Canada

E-mail: glbryson@ottawahospital.on.ca

\section{References}

1 Lui AC, Polis TZ, Cicutti NJ. Densities of cerebrospinal fluid and spinal anaesthetic solutions in surgical patients at body temperature. Can J Anaesth 1998; 45: 297-303.

2 Ben-David B, Miller G, Gavriel R, Gurevitch A. Lowdose bupivacaine-fentanyl spinal anesthesia for cesarean delivery. Reg Anesth Pain Med 2000; 25: 235-9.

3 Gaiser RR, Cheek TG, Gutsche BB. Comparison of three different doses of intrathecal fentanyl and sufentanil for labor analgesia. J Clin Anesth 1998; 10: 488-93.

4 Dahl JB, Jeppesen IS, Jorgensen H, Wetterslev J, Moiniche $S$. Intraoperative and postoperative analgesic efficacy and adverse effects of intrathecal opioids in patients undergoing cesarean section with spinal anesthesia: a qualitative and quantitative systematic review of randomized controlled trials. Anesthesiology 1999; 91: 1919-27.

\section{Diagnosis of brain death with the electroencephalogram}

To the Editor:

In a recently published, single-case study, Rimmelé et al. ${ }^{1}$ claim that the electroencephalogram (EEG) is not an adequate test to confirm the diagnosis of brain death. Since additional angiography disclosed residual cerebral blood flow (CBF), it was concluded that electrocerebral silence was misleading and, although clinical criteria were fulfilled, the patient was not assessed as being brain dead. I submit that the authors' conclusions are not warranted.

The patient was examined neurologically and two EEGs were recorded shortly after injection of etomidate, $20 \mathrm{mg}$. The half-life of etomidate is 0.5 to $1.25 \mathrm{hr}$ and that of its metabolites is $4.5 \mathrm{hr}$, so it is not appropriate to state that any sedation was absent. Usually we measure plasma levels to ensure they are below therapeutic range or we wait four times the longest half-life. Here, clinical examinations, as well as EEGs, may still have been influenced by sedatives.

Apparently, the movement of the right arm was spinal - as the authors themselves admit - and did 